Dan Valeriano F. Daffon, MD

Alberto F. Calderon, MD

Francisco A. Victoria, MD

Department of Otorhinolaryngology Head and Neck Surgery

Ospital ng Maynila Medical Center
Correspondence: Dr. Dan Valeriano F. Daffon

Department of Otolaryngology-Head and Neck Surgery Ospital Ng Maynila Medical Center

Quirino Ave. corner Roxas Blvd., Malate, Manila 1004 Philippines

Phone: (632) 524-6061 local 220

Email:ommc_enthns@yahoo.com

Reprints will not be available from the author

The authors declared that this represents original material that is not being considered for publication or has not been published or accepted for publication elsewhere in full or in part, in print or electronic media; that the requirements for authorship have been met by each author, and that each author believes that the manuscript represents honest work.

Disclosures: The authors signed disclosures that there are no financial or other (including personal) relationships, intellectual passion, political or religious beliefs, and institutional affiliations that might lead to a conflict of interest.

\section{Nasal Glial Heterotopia: Unsuspected Brain Tissue in the Nasopharynx}

\begin{abstract}
Objective: To report a case of ectopic brain tissue in the nasopharynx; discuss the differential diagnoses for and management of, this unilateral nasal mass in a pediatric patient, and describe the diagnostic difficulties and eventual treatment.
\end{abstract}

\section{Methods:}

\author{
Study Design: Case Report \\ Setting: Tertiary Government Hospital \\ Participant: One
}

Results: A 13-year-old boy consulted with an obstructing mass in the nasopharynx and severe muscle wasting, weight below his age and concurrent anemia. CT-scan revealed a soft-tissue mass occupying a maxillary sinus and nasopharynx consistent with an antrochoanal polyp. Initial biopsy revealed a papilloma, but the clinical picture warranted preoperative preparations for a possible vascular tumor such as juvenile angiofibroma. Transpalatal excision and final histopathology yielded nasal glial heterotopia.

Conclusion: When confronted with a unilateral nasal mass in a pediatric patient, aside from the usual considerations, embryologic anomalies particularly those of the intracranial protrusion variety should be strongly considered. Scrupulous preoperative evaluation and sufficient diagnostic modalities must be pursued in order to arrive at a correct diagnosis, treatment plan and prevent possible complications especially those related to intracranial communication.

Keywords: nasal glioma, glial heterotopia, congenital nasal masses

Tumors in the nasal cavity are rare in the pediatric age group. Althought most are benign, they can cause serious problems in children. These lesions may arise from an inflammatory cause, neoplastic process or alteration during embryonic development. Usually evident during infancy, some may remain unrecognized until late childhood. The diversity of presentation may not point to a specific cause, making it cumbersome for clinicians to diagnose. We report the case of a teenage boy who was diagnosed with, and managed for, a vascular tumor that later turned out a totally different entity. 


\section{CASE REPORT}

A 13-year-old boy was brought to us due to nasal obstruction of two years' duration diagnosed as rhinosinusitis. He was prescribed unrecalled medications affording little relief. He tolerated the condition for another year as it progressed to a right unilateral nasal obstruction with associated episodes of epistaxis. His mother also noted a bulge in the soft palate but they did not consult at this time.

A few months later, the mother noted hyponasal speech and a fleshy mass protruding into the oropharynx. The mass rapidly increased in size until it occupied half of the oropharynx, making it visible upon mere mouth-opening.

Marked weight loss with episodes of loud irregular snoring and subsequent nocturnal apneic episodes frequently disrupting sleep ensued. The boy exhibited a typical "skin and bone" appearance and increasingly became a mouth breather. Persistent weakness prompted the family to finally consult at our institution.

On physical examination, the boy was noted to be cachectic (Figure 1A) with Body Mass Index of $10.5 \mathrm{~kg} / \mathrm{m}^{2}$, severe muscle wasting and weight below his average age group. He also had a resting open-mouth facies and dull facial appearance. Otolaryngologic examination was unremarkable except for peeled-grape-like mass in the right nostril on anterior rhinoscopy. There was also bulging of the soft palate and a fleshy mass protruding into the oropharynx. (Figure 2A) With an impression of antrochoanal polyp, a CT-Scan revealed a soft-tissue density filling the right maxillary sinus with complete obstruction and widening of the osteomeatal unit, consistent with a large polyp. (Figure 3)

The boy also developed anemia with haemoglobin at $8 \mathrm{mg} / \mathrm{dl}$, prompting admission and transfusion of 1 unit of packed red blood cells. On biopsy of the intranasal and oropharyngeal mass, the latter bled easily on minimal manipulation. Fearing a vascular tumor, punch biopsy of the oropharyngeal mass was deferred. Histopathologic

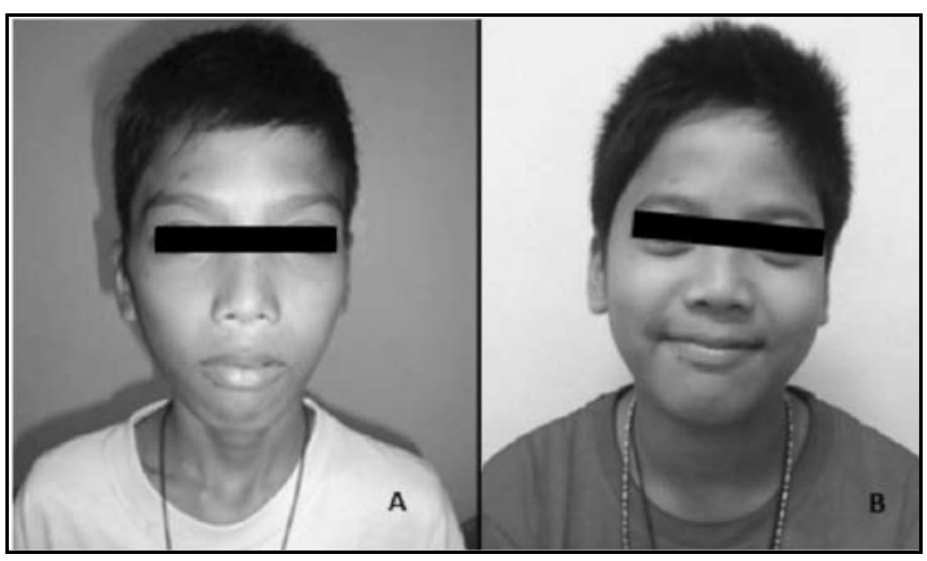

Figure 1. Profile picture of the patient: A. at initial consult B. 6 months post-operatively
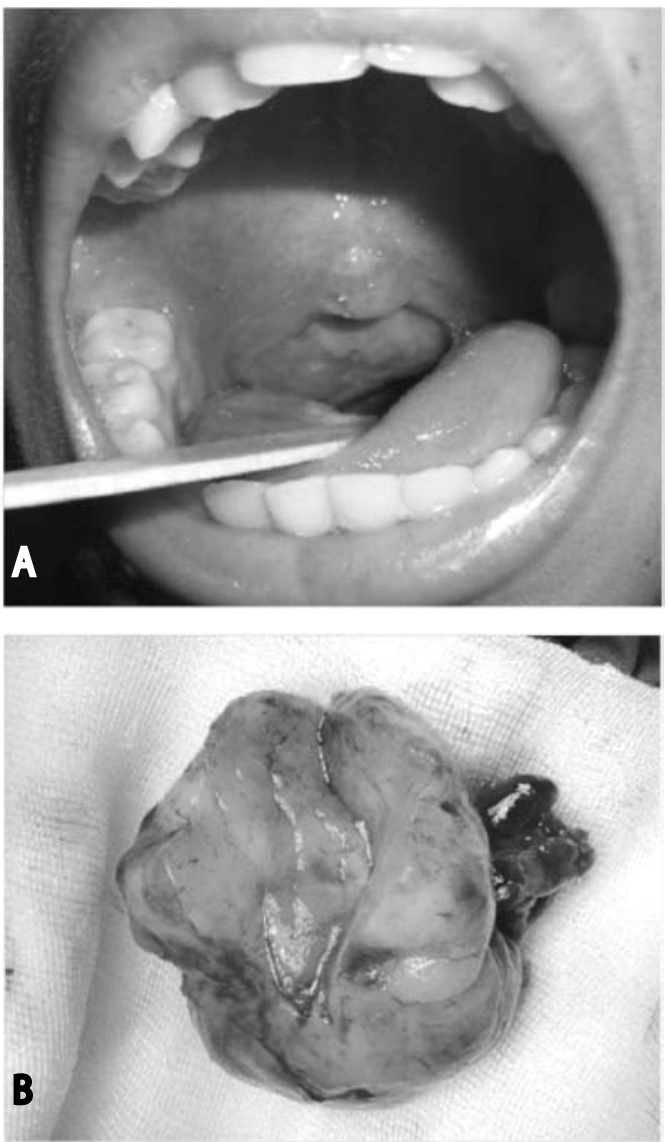

Figure 2. A. pre-operative oropharyngeal view revealing firm, solid nasopharyngeal mass protruding into oropharynx (left); and B. excised mass with gel-like consistency (right)

examination of the intranasal specimen revealed an edematous stroma infiltrated with chronic inflammation lined with ciliated columnar epithelial cells exhibiting mild dysplastic and koilocytic changes consistent with a nasopharyngeal papilloma.

Because the clinical picture was inconsistent with this result, juvenile angiofibroma was presumptively diagnosed and a definitive plan for excision begun. A contrast CT-scan was deferred due to history of allergy. Following caloric augmentation and with four units of fresh whole blood prepared, transpalatal excision was performed. Intraoperatively, the mass was noted to be attached to the superior aspect of the posterior border of the septum. Blunt dissection easily freed it from surrounding structures. Grossly it measured $5 \times 6 \mathrm{~cm}$, was firm, whitish and well encapsulated with a gel-like consistency. (Figure 2B) Estimated blood loss was 500cc. Final histopathologic report revealed a mixture of mature astrocytes, glial fibers and fibrovascular connective tissue with a mild to moderate degree of fibrosis. There was no evidence of malignancy in all sections. (Figure 4) The final diagnosis was Nasal Glial Heterotopia. 

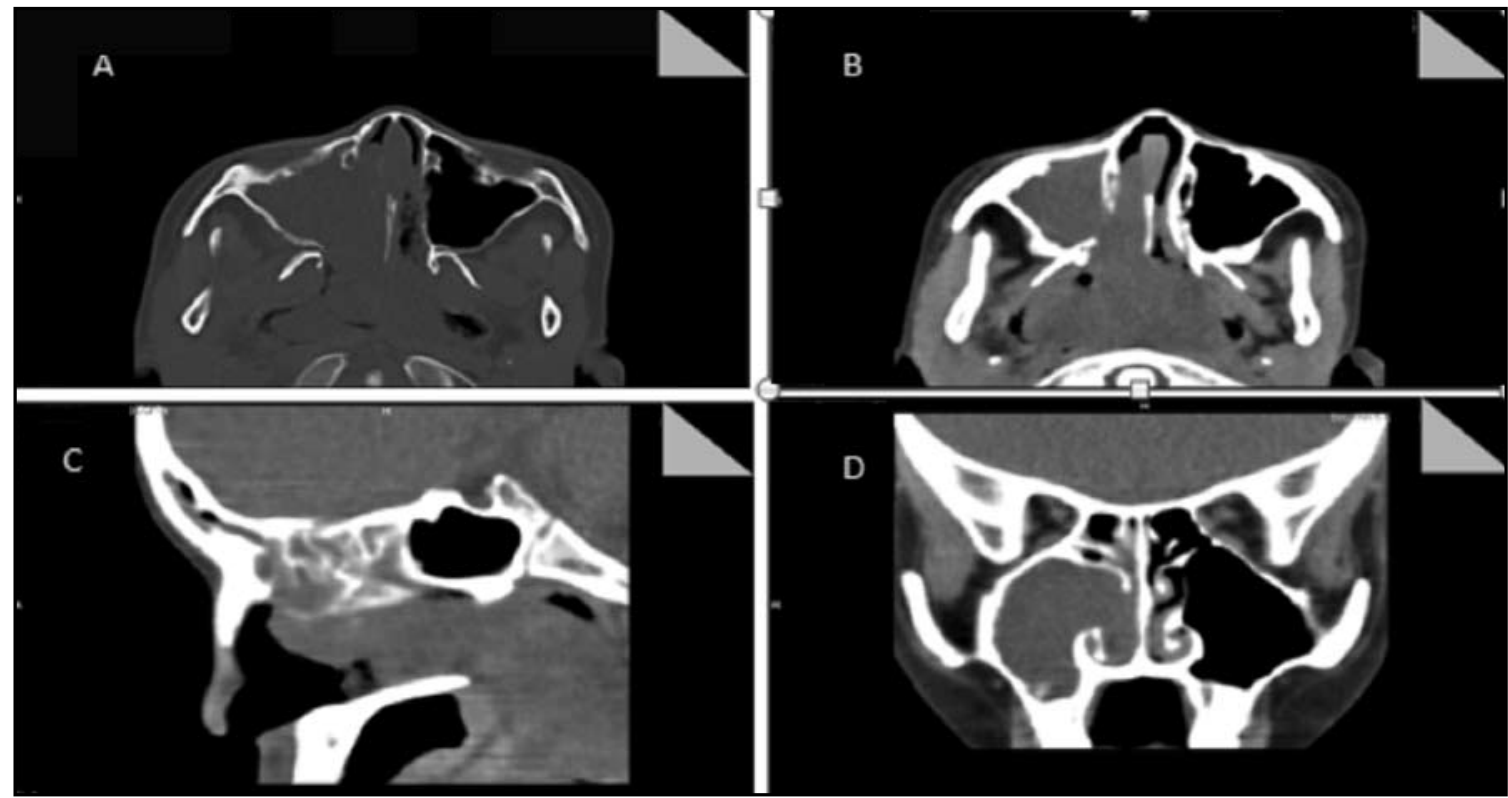

Figure 3: PNS CT-Scan: A. axial view (bone window) B. soft tissue density in the right maxillary sinus, mid to posterior aspect of the right nasal cavity and completely obstructing the nasopharyngeal airway C. sagittal view showing bony destruction of the right medial maxillary wall D. coronal view with completely obstructed and widened right osteaomeatal unit and patent left osteomeatal complex.

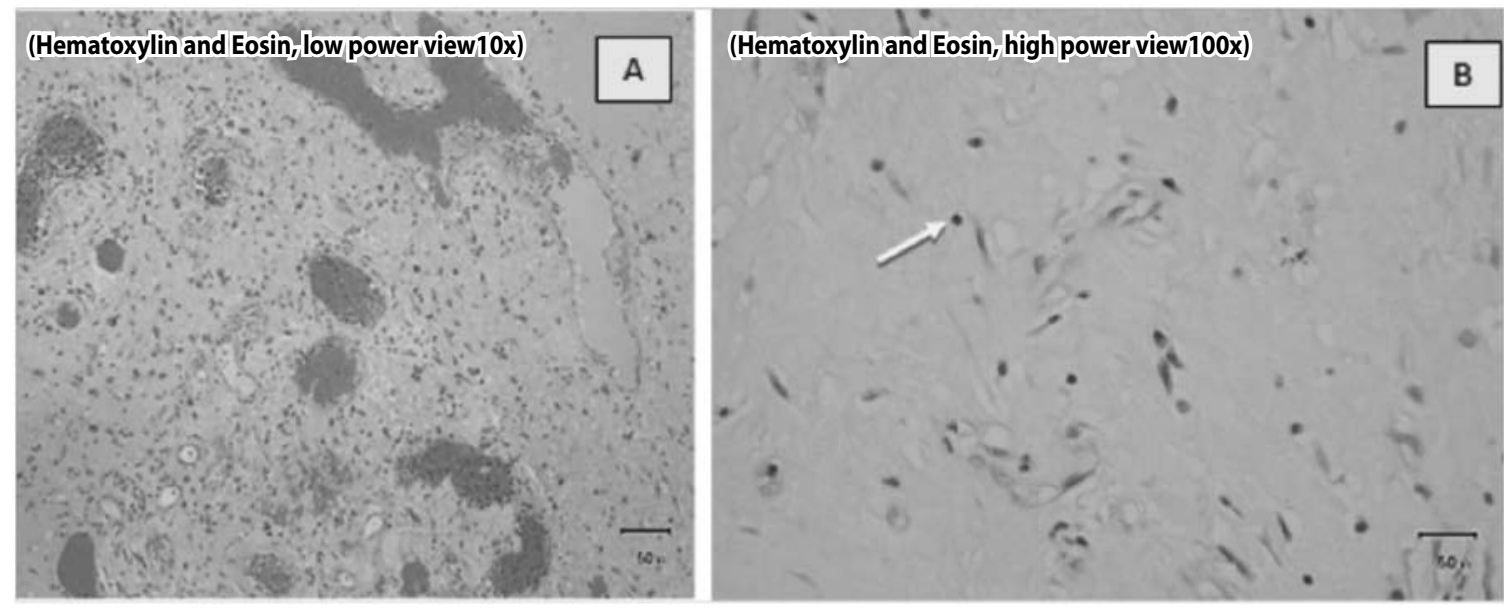

Figure 4: Final histopathologic slides A. low power view (10x) B. high power view (100x), Hematoxylin and Eosin stain showing a mixture of mature astrocytes, glia fibers (arrow) and fibrovascular connective tissue with a mild to moderate degree of fibrosis.

\section{DISCUSSION}

A unilateral nasal mass in a pediatric patient should be regarded with a high level of suspicion even if it presents like a mere polyp (peeled-grape appearance). Differential diagnoses should include vascular neoplasm (specifically juvenile angiofibroma), papilloma and even malignancy-- and because of the age of the patient, embryologic anomalies.

The rarity of nasal glial heterotopia as an embryologic anomaly is interesting but the twists and turns that followed the several changing impressions in managing this case made it even more interesting.

The marked weight loss of this patient could be attributed to dysphagia from oropharyngeal obstruction, decreased appetite from anosmia of nasal obstruction, biopsychophysiologic consequences of chroinic illness (including the despair of isolation from friends, school absences and altered activities of daily living) as well as malignancy.

With the peeled-grape appearance of this unilateral mass and the plain CT-scan results, it would have been easy to dismiss this as an antrochoanal polyp and proceed with endoscopic polypectomy. In hindsight, this would have been successful considering the narrow attachment to the posterior aspect of the nasal septum.

With age, unilaterality and size of the mass factored in, prudence overcame the initial plan in favor of a biopsy of the anterior and oropharyngeal components. However, bleeding forestalled biopsy of 
CASE REPORTS

the latter. The histopathologic reading of "nasopharyngeal papilloma" for the former was incongruent with the nature and behavior of the mass. The thought of a male teen with a bleeding unilateral nasal mass made it extremely difficult not to consider a juvenile angiofibroma. After all was said and done, the mass turned out to be an embryologic nasal glial heterotopia.

Nasal glial heterotopia or "nasal glioma" represents heterotopic or "ectopic" mature glial tissue found in or around the nose. 'Although the term implies a neoplasm, it is not a true neoplasm but a collection of normal tissue found in an abnormal location, or "choristoma." In essence it is a congenital malformation with a male to female predominance of $3: 1$. This rare congenital anomaly is estimated to occur in $1: 20,000$ to 40,000 births. ${ }^{1}$

How can brain tissue present in the nasal cavity with an intact skull base? This results from alterations in embryologic events that produce the nose, frontal-basal skull and intracranial contents. Such alterations include faulty closure of the anterior neuropore with defects in the fonticulus frontalis, foramen caecum, cribiform plate or sphenoid and ethmoid bones. Gliomas or rests of neuroglial tissue result when brain tissue is isolated extracranially by the fusion of cranial sutures without the inclusion of meninges although a fibrous stalk may persist. Hence, they remain unsuspected in the nasal cavity. ${ }^{2}$

About $30 \%$ of nasal gliomas are found intranasally, $60 \%$ extranasally, and only $10 \%$ in both locations. ${ }^{3}$ Intranasal lesions usually present with nasal obstruction or nasal deformity, ${ }^{1}$ or may present as mass protruding from a nostril, or more frequently, as a pale, glistening, polypoid mass within the nasal cavity or nasopharynx as seen in this patient. ${ }^{2}$ Rarely, there is epistaxis or spontaneous cerebrospinal fluid leakage. ${ }^{2}$ Extranasal gliomas are firm, incompressible masses that often occur along the nasomaxillary suture or near the glabella. They do not transilluminate or expand with Valsalva or crying (Furstenberg test). ${ }^{4}$ The overlying skin may have telangiectasia and they may easily be confused with haemangiomas. The nasal bridge may be broadened and the space between the eyes may be widened. ${ }^{2}$ They are differentiated from nasal encephaloceles which always have a dural connection, transilluminate and have a positive Furstenberg test. ${ }^{5}$

Although rare, these are clinically important because of the potential for intracranial connection. Overall, only 10 to $15 \%$ of gliomas have a connection to dura. Intranasal gliomas are two to three times as likely to have such a connection compared to their external counterparts. ${ }^{5}$ Therefore, office biopsies should be withheld without prior imaging studies and incisional biopsies should be performed with caution, if at all, under controlled circumstances in the operating room due to the risk of serious cerebrospinal fluid leak with subsequent intracranial infection risk. $^{3}$

Histologic examination reveals neuroglial tissue (astrocytes and connective tissue) without a true capsule. ${ }^{5}$ Neurons are usually absent. Rarely, choroid plexus, ependyma-lined clefts and pigmented retinal epithelium are seen especially those of the palate and nasopharynx. The glial tissue can be confirmed by immunoreactivity for glial fibrillary acidic protein (GFAP) or $\mathrm{S} 100$ protein. $^{1}$

The possibility of nasal glioma may be entertained based upon clinical evaluation and the use of adjunctive studies such as radiographic imaging scans (CT and MRI). These may also reveal a soft tissue mass without an intracranial component or bony defect in the floor of the anterior cranial fossa tissue. Even with high-resolution computed tomography and magnetic resonance imaging, the connection may be very small and unapparent. ${ }^{3}$ Definitive diagnosis is often only possible after complete surgical excision. ${ }^{3}$

The preferred management for nasal glioma is surgical excision. If there is no evidence of intracranial communication external/ transfacial approaches are adequate. Neurosurgical consultation may still be necessary if a previously unrecognizable tract is identified intraoperatively to be coursing to the skull base. ${ }^{2}$ Intranasal gliomas, if small and lacking intracranial communication may be excised endoscopically.

Though this lesion has a slow growth rate and is benign without any potential for malignant degeneration, delays in treatment may lead to distortion of the septum and nasal bone, or infection. Overall, recurrence rates of 4 to $10 \%$ have been reported. ${ }^{6}$

Our patient had an uneventful recovery and regained an adequate body mass index with normal physical examination findings and no noted recurrence at six months. (Figure 1B)

When confronted with a unilateral nasal mass in a pediatric patient aside from the usual considerations, embryologic anomalies particularly those of the intracranial protrusion variety should be strongly considered. Scrupulous preoperative evaluation and sufficient diagnostic modalities must be pursued in order to arrive at a correct diagnosis, treatment plan and prevent possible complications especially those related to intracranial communication.

REFERENCES

1. Kindblom LG, Angervall L, Haglid K. An immunohistochemical analysis of S-100 protein and glial fibrillary acidic protein in nasal glioma. Acta Pathol Microbiol Immunol Scand A. 1984 Sep; 92(5): 387-389.

1. Hirsh LF, Stool SE, Langfitt RW, Schut L. Nasal glioma. J Neurosurg. 1977 Jan; 46(1): 85-91,

2. Burkey B, Koopmann CF, Brunberg J. The use of biopsy in the evaluation of pediatric nasopharyngeal masses. Int J Pediatr Otorhinolaryngol. 1990 Nov; 20(2): 169-179.

3. Ajose - Popoola O, Lin HW, Silvera VM, Teot LA, Madsen JR, Meara JG, Rahbar R. Nasal glioma: prenatal diagnosis and multidisciplinary surgical approach. Skull Base Rep. 2011 Nov; 1(2): 8388.

4. Hodges FJ. Clinical evaluation and diagnosis of tumors of the paranasal sinuses and nasal cavity. In:Thawley S, Panje W, Batsakis J, Lindberg R, editors. Comprehensive management of head and neck tumors. Philadelphia, PA:WB Saunders; 1999. p. 511-18.

5. Magit AE. Tumors of the nose, sinuses and nasopharynx. In: Bluestone CD, Stool SE, Alper $C$ Arjmand E, Casselbrant M, Dohar J, Yellon R, editors. Pediatric otolaryngology. 4th ed. Philadelphia, PA:WB Saunders; 2003. p. 1053-64. 